\title{
Maps under the global condition: a new tool to study the evolution of cartographic language
}

\author{
Cherrier Pierre $^{\mathrm{a}, *}$, Lentz Sebastian ${ }^{\mathrm{a}}$, Moser Jana ${ }^{\mathrm{a}}$, Pflug Laura ${ }^{\mathrm{a}}$

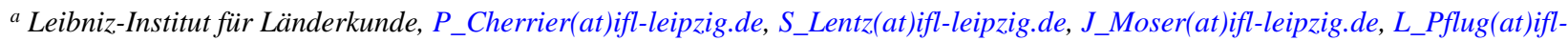 \\ leipzig.de
}

Keywords: History of Cartography, Atlases, Thematic Maps, Atlas Analysis, Quantitative Map Analysis

Introduction

Maps are a means of communication with their own language. This contribution makes a methodological proposal for a tool for to analyse the cartographic language of thematic maps and atlases. Based on the work of Jacques Bertin and on approaches of the Visual Studies, this methodology works on decoding maps in terms of their basic elements, the signs and graphic objects that compose them. As a tool it should allow comparative research on cartographic productions, both, synchronically and diachronically. It suggests two analytical schemes, one for maps and the other for complex map-editions, e.g. atlases.

On the example of spatial entities (state territories, natural areas etc.), the first part of this contribution introduces the semiotic analysis-scheme for thematic maps. It shows how to deal systematically with signs, signatures and graphic objects on maps. Such analyses should produce the fundament for comparative approaches, which allow to detect typical patterns in cartography and to identify elements of cartographic languages.

We are interested in the cartographic languages of maps used in atlases. To do this we have chosen a quantitative analysis of the visual content, maps, diagrams and images. The quantitative method makes it possible to analyse a large corpus of maps and atlases, thus making it possible to make comparisons between contents both diachronically and synchronically, i.e. comparisons in time and space. This is an approach relatively rarely used in cartography. There are few studies that produce a quantitative analysis of cartographic content. Among the existing ones, that of Alexandre Kent and especially that of Muelhenhaus on the Goode atlas series. We are following in the footsteps of these studies. To do this, we decided to adopt a semiological approach to the study of maps. Of course, we cannot talk about maps and semiology without mentioning Jacques Bertin and his book: graphic semiology: diagrams, networks, maps (1963) in which he tried to define a "grammar" by establishing rules of good cartographic practice, even if the book is not exclusively reduced to the map.

The book itself does not contain any reference, but it can be said that graphic semiology is itself derived from linguistic semiology, developed in particular by Ferdinand Saussure. However, although Bertin's work has influenced many cartographers in the design of maps, the method has been little used in the cartographic analysis itself. Semiology is an approach that has been used mainly in the analysis of images and diagrams rather than in cartography. Although it is true that iconographic analysis studies in semiology claim more Barthes and Saussure than Bertin.

The map can also be considered as an image. Several iconographic analysis studies have thus integrated the map as an object of study. This is the case, for example, of engelhardt who, in his thesis "the language of graphics: a framework for the analysis of syntax and meaning in maps, charts and diagram" (2002), focuses on several types of iconography, even if the map remains a central element of his analysis. Another example is the work of André Lavarde, who in his article "la flèche : le signe qui anime les schémas" (1996) focuses on the history of the use of the arrow in diagrams, while evoking its use in geographical maps. There are therefore bridges between iconographic and cartographic analysis.

This research is therefore a continuation of the work of Bertin, Mulhenhaus and to a certain extent Engelhardt. The coding system we have developed for our cartographic analysis is divided into three parts and divided tehemselves into several categories. Each category corresponds to a column in the table. From there, there are two ways to fill in the columns. In the first case by filling in the field with the requested information such as the title of a map. Or in a second case to enter 0 ; 1 ; or 2 depending on whether the information that corresponds to the absence, presence or uncertainty of the requested information. So if the map coded uses the Mercator projection then it will be entered 1 in the column "map projection: cylindrical projection" and 0 in the column "map projection: compromise". 
The table is composed of three parts. The first part concerns the general information of the coded map (image 1). This is for example the name of the atlas, the page, the chapter in which the map is located. Then more general information about the map itself is coded like for example its title, theme, scale, type of projection used, etc. This makes it possible to collect a set of basic data. It should be noted that, as mentioned above, we do not only code maps but also other forms of visual representations of space that can be found in atlases. For example, there are images, satellite photos or diagrams that can represent different geographical areas. If the coded object is not a map, this is specified. There is a category provided for this purpose. When coding, cartography-specific elements, such as map projection, are therefore not taken into account. Not all the columns in our table are intended to be filled by each map or coded image. The codification process is therefore flexible. Although the code does not focus only on maps, they represent the vast majority of the content of the atlases studied. This is why we refer more to the "map" rather than to the "visual representation of space". However, even if they are in the minority, it is important in the analysis to take into account representations of space other than cartography.

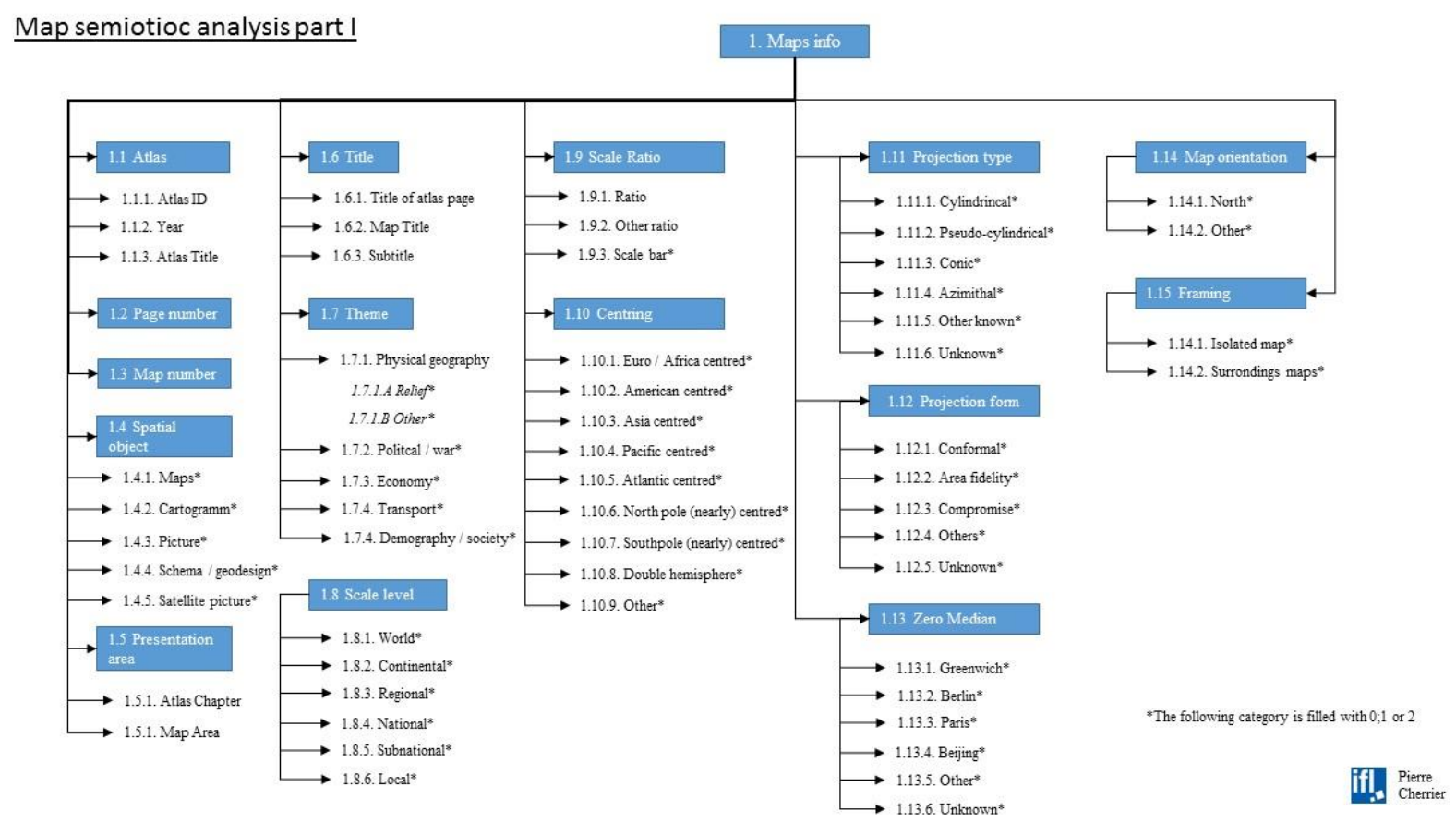

The second part of the table focuses on the signs used by the maps. First of all, we have chosen to divide them into three categories: symbols that are related to the point of the line and the surface. These are the three elementary figures of geometry that Bertin calls implantations. It is from these three types of locations that the different symbols are created. We have distinguished them between the thematic symbols, which are there to illustrate the theme of the map, to convey its message and the Background symbol present to help the reader to orientate himself in space. This is the case, for example, of the equator's path, which is rarely thematic, but rather serves as a geographical point of reference. Of course, the thematic symbols vary according to the theme of the map. Thus, territorial borders can be considered thematic if it is a political map, but will be considered Background information if it is present on a map representing global forest cover. The purpose of this part is to have as much content as possible on the elements that make a map. 

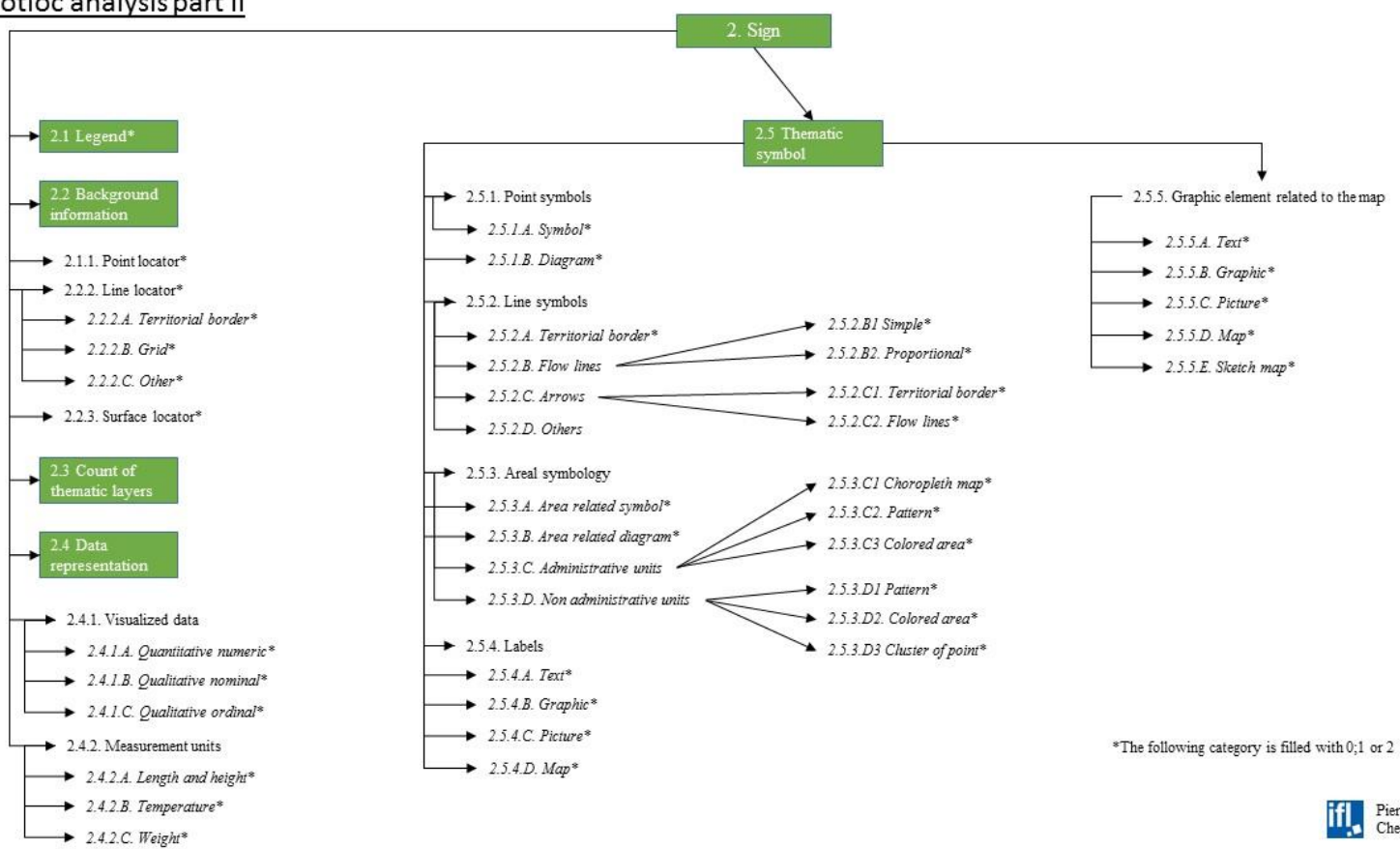

ifil. $\begin{aligned} & \text { Pierre } \\ & \text { Cherrier }\end{aligned}$

The third and last part of the table refers to visual variables. To be interested in visual variables is to be interested in the interactions between symbols. It is on this part that we rely most on Bertin's work. We have thus taken 5 of the 7 variables he defined. The orientation and the two dimensions of the plan were excluded from our study because they are constant in the cartographic production. It would therefore be irrelevant to record them each time. This is not the case for the remaining components: size, value, texture, shape, and colour. These are elements that may be present in cartography but are not individually necessary. These visual variables form the basic grammar of the "cartographic language". Studying the visual variables is a way for us to observe how the different signs interact with each other and to see how an information is convey. These visual rules have been established in the 1960s, therefore it the relevance of using this framework to study historical map can be questioned. But Bertin did not design his rules from scratch, he relied on previous mapping practices. It is therefore interesting to observe how often they have been used.

\section{Map semiotioc analysis part III}

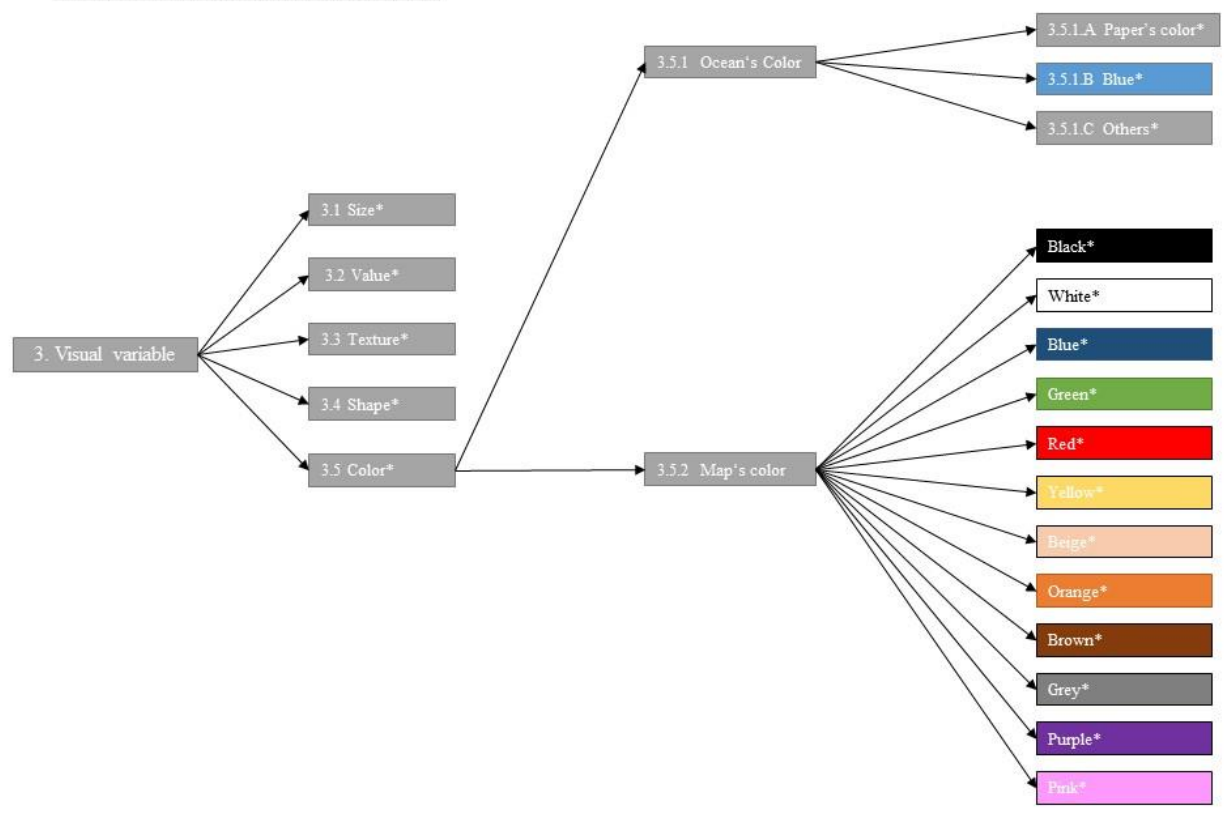


The second part deals with map themes and regional structures of atlases. Using principles of Visual Studies, it suggests to observe atlases as a whole as cultural products, each subject to a visual programme that determines the frameworks of its expressions and its claim for representativeness. By comparing elements like projection, scale, maps-themes, regional sequences etc. systematically, one may unveil the specific interpretations of world views which are contained in the atlas' concepts. As some atlases are published in a long series of editions, they become interesting research objects in an evolutionary perspective.

In a diachronic perspective the coding scheme suggested here, focussing themes and regional subdivisions of atlases, builds the fundament for longitudinal studies. Both methodological parts should make cartographic and atlas-studies more compatible to cultural and historical research approaches.

Taking the example of a few maps from French atlases from nineteen centuries to the early 2000s the second part of this contribution wants to give an idea, how this methodology can be used to study the evolution of cartographic language over time under the influence of the global condition and how French cartographers faced the challenge of representing a growing interconnected world and which graphical tools they developed. 